Voix et Images

voixetimages

\title{
Bibliographie de Jack Kerouac
}

\section{Rod Anstee, Maurice Poteet et Hélène Bédard}

Volume 13, numéro 3 (39), printemps 1988

Jack Kerouac et l’imaginaire québécois

URI : https://id.erudit.org/iderudit/200732ar

DOI : https://doi.org/10.7202/200732ar

Aller au sommaire du numéro

Éditeur(s)

Université du Québec à Montréal

ISSN

0318-9201 (imprimé)

1705-933X (numérique)

Découvrir la revue

Citer ce document

Anstee, R., Poteet, M. \& Bédard, H. (1988). Bibliographie de Jack Kerouac. Voix et Images, 13(3), 426-434. https://doi.org/10.7202/200732ar d'utilisation que vous pouvez consulter en ligne.

https://apropos.erudit.org/fr/usagers/politique-dutilisation/ 


\title{
Bibliographie de Jack Kerouac
}

\author{
par Rod Anstee, Ottawa, \\ Maurice Poteet, Université du Québec à Montréal et \\ Hélène Bédard, Université du Québec à Montréal
}

\section{a) Kerouac en français}

Cette liste d'ouvrages de Jack Kerouac traduits en français a été compilée par l'archiviste Rod Anstee d'Ottawa. Elle vise à corriger certaines erreurs qui persistent dans des bibliographies connues (par exemple, le nom du traducteur de Sur la route est souvent mal écrit; certains ouvrages «traduits» n'existent pas; certains ne sont que partiellement traduits, etc.). En même temps, cette liste précise les «premières éditions» de Kerouac en français, tout en donnant des détails concernant les réimpressions, renseignements surtout intéressants pour les collectionneurs et les chercheurs. Il faut souligner que Pic est le seul ouvrage de Kerouac a avoir été traduit au Québec. Ce travail est l'œuvre de Daniel Poliquin qui traduit actuellement le premier roman de Kerouac, The Town and the City (sortie prévue en 1989 chez Québec/Amérique). L'édition française (européenne) de Pic a paru aux éditions de la Table ronde, à Paris, en janvier 1988, avec glossaire.

Anstee précise que la plupart de ces renseignements proviennent directement des maisons d'édition, grâce à l'aide de Louis Dupont, de Guy Marchand, de Christian Bourgois, de Jean-Marc Roberts (Seuil), de Bernard Delaville (Seghers) et surtout de François Laurent (Gallimard). Pour tout autre information, s'adresser à Rod Anstee, 1045, Cahill Drive West, Ottawa (Ontario), K1V 9J1.

\section{TITRE}

1. Sur la route

(On the Road, 1957)

Tr. Jacques Houbart ${ }^{1}$

\section{MAISON D'ÉDITION DATE}

Gallimard

( +42 copies imprimées sur papier spécial)

Folio $\mathrm{n}^{\circ} 61$
Folio $\mathrm{n}^{\circ} 766$
Folio $\mathrm{n}^{\circ} 766$ (nouv. couv.)

10 mars 1972

28 juin 1982

12 janvier 1987

19 octobre 1962

Gallimard

19 février 1960

2. Docteur Sax 
(Doctor Sax, 1959)

Tr. Jean Autret
(+ 41 copies imprimées sur papier spécial)

3. Les Clochards célestes Gallimard

21 août 1963

(Dharma Bums, 1958)

( +26 copies imprimées sur papier spécial)

Tr. Marc Saporta

Folio $\mathrm{n}^{\circ} 565$

2 avril 1974

4. Les Souterrains

Gallimard juillet 1964

(The Subterraneans, 1958)

( +26 copies imprimées sur papier spécial)

Tr. Jacqueline Bernard

Folio $n^{\circ} 1690$

5 novembre 1985

5. Big Sur

Gallimard

25 septembre 1966

(+ 27 copies imprimées sur papier spécial)

Tr. Jean Autret

Folio $n^{\circ} 1094$

12 septembre 1979

Folio $n^{\circ} 1094$ (nouv, couv.) 18 avril 1985

6. Les Anges vagabonds

Denoël

1er trimestre 1968

(Desolation Angels, 1965 , = «Passing

Through ${ }^{2}$ )

Tr. Michel Deutsch

(Aux Lettres nouvelles)

20 septembre 1973

7. Le Vagabond solitaire Gallimard

10 juin 1969

(Lonesome Traveler, 1960)

Tr. Jean Autret

( +26 copies imprimées sur papier spécial)

Folio $n^{\circ} 1187$

6 mai 1980

8. Satori à Paris

Gallimard

8 février 1971

(Satori in Paris, 1960) («Sous jaquette illustrée»)

Tr. Jean Autret

9. Visions de Gérard Gallimard

25 février 1972

2 Le livre un, «Desolation Angels», n'existe pas en français. 
(Visions of Gerard, («Sous jaquette illustrée») 1963)

Tr. Jean Autret

10. Mexico City Blues

Christian Bourgois

(2 volumes)

2e trimestre $1976 /$

(Mexico City Blues,

Union générale $\ll 10 / 18 »$ 1959)

Tr. Pierre Joris

4e trimestre 1976

$4^{e}$ trimestre 1978 /

2e trimestre 1979

11. Poèmes

Seghers

1976

(Scattered Poems, 1971)

Seghers «Autour du monde»

$1^{\text {er }}$ trimestre 1978

Tr. Philippe Mikriammos

12. Le Livre des rêves

Flammarion

février 1977

(Book of Dreams, 1960)

Tr. Anne-Christine Taylor

13. L'Écrit de l'éternité d'or

(Scripture of the

Golden Eternity, 1960)

Tr. Philippe Mikriammos

14. Vanité de Duluoz

Christian Bourgois

17 juillet 1979

(Vanity of Duluoz, 1960)

La Différence

12 mars 1979

Tr. Brice Matthieussent

15. Tristessa

Stock

14 octobre 1982

(Tristessa, 1959)

Québec/Amérique

janvier 1983

Tr. Catherine David

Stock

9 janvier 1984

16. Maggle Cassidy

Québec/ Amérique

avril 1984

(Maggie Cassidy, 1959)

Seuil «Point»

septembre 1984

Tr. Béatrice Gartenberg

Québec/Amérique

«Espace»

$2^{e}$ trimestre 1987

17. Pic

Québec/Amérique

4e trimestre 1987

(Pic, 1971)

La Table ronde

«Miroir de la terre»

janvier 1988

Tr. Daniel Poliquin 
18. (Titre français à venir) Québec/Amérique (The Town and the City, 1950)

Tr. Daniel Poliquin à paraître en 1989

La Table ronde «Miroir de la terre»

à paraître en 1989

b) Écrits en français sur Kerouac et son œuvre (1963-1987)

1987

[ANONYME], «Kerouaciens de tous pays...», Qui-Vive international, février 1987.

AGOSTINI, Bertrand, «Jack Kerouac: 13 haiku», Filigrane, vol. II, mars 1987.

WADDELL, Eric, «Kerouac à Québec, les 1, 2, 3 et 4 octobre prochain!», N'importe quelle route, vol. $1, \mathrm{n}^{\circ} 1$, mars 1987.

ROYER, Jean, «Sur les traces de Jack Kerouac», le Devoir, 4 avril 1987, p. D2.

[ANONYME], «Québec, 1-2-3-4 octobre 1987, La Rencontre internationale Jack Kerouac: une invitation à pénétrer dans l'Univers kerouacien», Bulletin de la société canadienne d'études ethniques, vol. XIV, n² 2, été 1987.

[ANONYME], «Rencontre Jack Kerouac», le Soleil, 12 juin 1987.

GIROUX, Jacques, «Rencontre Jack Kerouac», le Journal de Québec, 17 .juin 1987 , p. 34.

Annonce de la Rencontre internationale Jack Kerouac, octobre 1987, Québec Scope, vol, 1, $\mathrm{n}^{\circ}$ 6, juillet 1987.

DICKSON, Robert, «Of books and men: Ti-Jean, Patrice, Robert et les autres...», Llaison, $n^{\circ} 44$, septembre 1987, p. 5-6. Entrevue avec Patrice Desbiens: écriture spontanée de Kerouac.

CHIASSON, Herménégilde, «Le Grand Jack: une lecture qui devient un film...», Liaison, $n^{\circ} 44$, septembre 1987 , p. 7-8.

PERREAULT, Robert B., «Un colloque bien réussi», Lialson, $n^{\circ} 44$, septembre 1987, p. 9-10. Huitième colloque annuel de l'Institut français du collège de l'Assomption, 13-14 mars 1987: influence de Kerouac sur certains écrivains franco-américains d'aujourd'hui.

STANTON, Julie, «Jack Kerouac, d'hier à demain», le Devoir, 22 septembre 1987, p. 13.

PRESSE CANADIENNE, «Sommet sur Jack Kerouac à Québec début octobre», la Presse, 22 septembre 1987, p. A14.

VOISARD, Anne-Marie, «Les fans de Jack Kerouac se réuniront à Québec, en octobre», le Soleil, 22 septembre 1987, p. B14. 
GIROUX, Jacques, «Un colloque international», le Journal de Québec, 23 septembre 1987, p. 20.

ROBERGE, Pierre, «L'ONF ressuscite Jack Kerouac», le Journal de Québec, 23 septembre 1987, p. 21.

«Au pays de Kerouac», le Solell, 24 septembre 1987, p. D10. Annonce d'un voyage au pays des ancêtres de Kerouac.

HAMANN, Jean, «Kerouac: la rencontre des deux Amériques», Au fil des événements-Université Laval, 24 septembre 1987, p. 5.

«Rencontre internationale Jack Kerouac», le Soleil, 1er octobre 1987. Programme du $1^{\text {ex }}$ octobre.

GAUDRAULT, LÉonce, «Jack Kerouac au Musée du Québec. Des images sur l'œuvre littéraire du Canuck», le Solell, 1er octobre 1987, p. B15.

VOISARD, Anne-Marie, «Rencontre internationale Jack Kerouac. Un happening qui débute avec éclat», le Soleil, 2 octobre 1987.

GAUDRAULT, LÉonce, «Un document attachant mais qui ne rend pas la grandeur du héros: "Le Grand Jack" du cinéaste Herménégilde Chiasson», le Solell, 2 octobre 1987, p. C2.

PRESSE CANADIENNE, «Une centaine de fans de Jack Kerouac sont au rendez-vous de Québec», la Presse, 3 octobre 1987, p. B8.

«Moi pis grand-papa (extrait de "Pic", inédit de Jack Kerouac)», le Devoir, 3 octobre 1987, p. D1.

[ANONYME], «Deux amis intimes de l'écrivain racontent d'émouvants souvenirs: Carolyn Cassady et Allen Ginsberg», le Soleil, 3 octobre 1987, p. C3.

«Rencontre internationale Jack Kerouac», le Soleil, 3 octobre 1987, p. C12. Programme du 3 octobre.

BAUCH, Hubert, «Remembering Jack Kerouac, Fans from Orient Gather with Figures from Past at Quebec City Festival», The Gazette, 3 octobre 1987.

[ANONYME], «Un destin différent pour un Kerouac québécois selon la biographe Ann Charters», le Soleil, 4 octobre 1987, p. C1.

MARTEL, Réginald, «La Rencontre de Jack Kerouac: réminiscences d'un temps où, dans les boîtes de Québec et Montréal, la poésie disputait l'air rare à la fumée», la Presse, 5 octobre 1987, p. A16.

VOISARD, Anne-Marie, «L'auteur Jack Kerouac continue de provoquer la polémique», le Soleil, 5 octobre 1987, p. B5.

MORIN, Marc, «Première rencontre internationale: accent aigu comme dans Kerouac», le Devoir, 6 octobre 1987. 
PERONCEL-HUGOZ, J.-P., «Kerouac reconnu par les siens», le Monde, 9 octobre 1987.

MORISSET, Jean-M., «L'éternel retour de Jack Kerouac», le Devoir, 29 octobre 1987.

LESSARD, Alain, «Des questions à la bienséance», Nuit blanche, $n^{\circ} 30$, décembre 1987, p. 10-11. Propos de Daniel Poliquin qui vient de traduire Pic de Kerouac.

FORTIN, Andrée, «Vision de Jack», Nuit blanche, $n^{\circ} 30$, décembre 1987, p. 32 36. Américanité et influence de Kerouac sur le Québec.

TARDIF, Richard, «Un écrivain sous influence», Nuit blanche, $n^{\circ} 30$, décembre 1987, p. $37-38$.

FORTIN, Andrée, «Pier Vittorio Tondelli. Une nouvelle image de l'Italie», Nuit blanche, $\mathrm{n}^{\circ} 30$, décembre 1987, p. 40-41. Influence de Kerouac sur les jeunes écrivains italiens.

KEROUAC, Jack, Pic, traduction de Daniel Poliquin, Montréal, Québec/Amérique, $4^{e}$ trimestre $1987,148 \mathrm{p}$.

\section{6}

[ANONYME], «Sur la route de Kerouac: du 22 au 24 août», le Devoir, 25 juillet 1986, p. 5.

PETROWSKI, Nathalie, "Qu'est-il arrivé à Jack Kerouac?: Festival international du nouveau cinéma et de la vidéo de Montréal», le Devoir, 21 octobre 1986, p. 19.

\section{4}

REY, Henri-François, «Journal de lecture», Magazine littéraire, $\mathrm{n}^{\circ} 205$, mars 1984, p. 60-61. Commentaire sur Maggie Cassidy.

BEAULIEU, Victor-Lévy, «Kerouac adolescent à Lowell», le Devoir, 2 juin 1984, p. 24.

BEAULIEU, Victor-Lévy, «Pour en finir avec la beat generation», le Devoir, 18 août 1984, p. 20.

\section{3}

ROYER, Jean, «Kerouac chez les anges de l'enfer», le Devoir, 12 février 1983, p. 19.

\section{2}

GAUDRAULT, Léonce, «Pèlerinage au pays de Kerouac», le Soleil, 24 juillet 1982, p. D2. 


\section{1}

ARCHAMB AULT, Gilles, le Voyage ur distrait, Montréal, Stanké, 1981. Roman sur le mythe de Kerouac.

\section{0}

Dossier sur la beat generation: Kerouac, Ginsberg, Burroughs, Magazine littéraire, $\mathrm{n}^{\circ} 157$, février 1980, p. 8-37.

SORRELL, Richard S. «L'histoire en tant que roman, le roman en tant qu'histoire. Le roman ethnique franco-américain de langue anglaise», Vie française, numéro spécial: Premier colloque de l'Institut français (Assumption College), mars 1980, p. 64-76. Entre autres: On the Road.

RICARD, François, «La vécriture de Jack Kerouac», Liberté, $n^{\circ} 128$, mars-avril 1980 , p. 85-90. Eléments autobiographiques dans la prose de Kerouac.

LEROUX, André, «Sur nos écrans. Heart Beat, Séquences, vol. 25, $\mathrm{n}^{\circ} 101$, juillet 1980 , p. $40-41$.

COPPENS, Patrick, «Choix de lecture», le Devoir, 20 septembre 1980, p. 24. Article portant sur Kerouac.

GUÉGAN, Gérard, «Mes oncles Giono, Simenon, Kerouac et compagnie», Tel Quel, n 85, automne 1980, p. 79-80.

KEROUAC-HARVEY, Raymonde, I'Album, Beauceville (Qc), L'Éclaireur limitée, 1980. Album de famille des Kerouac du Québec (partie portant sur Jack Kerouac; lettre écrite par celui-ci au révérend $G$. Lévesque à propos de ses racines québécoises).

\section{9}

INGLES, Louise, «Jack Kerouac, le “damn Canuck” de la Québécité», la Presse, 7 septembre 1979.

BÉGUIN, Louis-Paul, «Ti-Jean Kerouac», le Devoir, 31 octobre 1979, p. 6.

BEGUIN, Louis-Paul, «Kerouac, écrivain américain», le Devolr, 7 novembre 1979 , p. 6.

GILFORD, Barry et LEE, Lawrence, les Vies parallèles de Jack Kerouac, traduction par Brice Matthieussant de Jack's Book, Paris, Henri Veyrier, coll. «Off», 1979.

\section{8}

PIEILLER, Evelyne, «Une des voix du rêve américain», la Quínzaine littéraire, $\mathrm{n}^{\circ} 314$. 
STARER, Jacqueline, «Les écrivains beats et le voyage», Études anglaises, $n^{\circ} 68$ et Didier, 1977. Cette étude comprend une des meilleures bibliographies sur Kerouac, Cassady, Corso, Ferlinghetti, Ginsberg, Holmes, Kaufman, Lamantia, McCLure, Solomon, Welch et Whalen.

STARER, Jacqueline, «Chronologie des écrivains beats jusqu'en 1969», Paris, Didier, 1977.

BEAULIEU, Victor-Lévy, «L'illumination de Jack Kerouac», le Devoir, 19 mars 1977. Article portant sur Mexico City Blues, traduit par Pierre Joris pour Christian Bourgois, Paris 1976.

\section{6}

DOMMERGUES, Pierre, «L'aliénation dans le roman américain contemporain», Paris, Éditions, 18 octobre 1976, vol. 1. Références occasionnelles à Visions of Gerard.

CHARTIER, Armand B. «The Franco-American Literature of New England: A Brief Overview» (présenté en 1976 au Symposium sur la littérature ethnique à Texas Tech University, 28 p., avec bibliographie). Publié dans Ethnic Literature Since 1976: The Many Voices of America, par Wolodymyr T. Zyla et Wendell M. Aycock, éditeurs, Lubbock (TX), Texas Tech Press, 1978, p. 193215.

THERRIER, Michel, «Béatitude et émerveillement dans "On the Road" de Jack Kerouac», Études anglaises, XXIX, $n^{\circ} 3$, p. 468-477.

1975

POUPART, Jean-Marie, «Rien de québécois chez le romancier Jack Kerouac», le Devoir, 8 février 1975, p. 16.

COMTOIS, Maurice, «Québécois, Américain, ou Canayen», le Devoir, 8 février 1975, p. 20.

CHARTERS, Ann, Kerouac, le vagabond, traduction par Monique Poublan de Kerouac, a Blography, Outremont, éditions l'Étincelle (en 1974, l'ouvrage de Charters était publié en France par Gallimard).

MARTEL, Réginald, «Kerouac: mourir de la fureur de vivre», la Presse, 8 février 1975 , p. 3.

TREMBLAY, Robert, «Jack Kerouac: le voyageur immobile», le Solell, 15 février 1975, p. D10.

LE PELLEC, Yves, «Visions of Cody ou Jack Kerouac, voyageur de l'Amérique», Caliban (Toulouse, France), $n^{\circ} 12$, p. 81-92. 


\section{$1973-1974$}

VAUTHIER, S. «Le Mexique et le mythe du voyage initiatique dans le roman de Jack Kerouac On the Road, Tilas, ns 13-14, p. 33-51.

THÉRIAULT, Jacques, «Les Anges vagabonds», le Devoir, 1er décembre 1973, p. 16.

TREMBLAY, Robert, «Les Anges vagabonds», le Soleil, 8 décembre 1973, p. 47.

LE PELLEC, Yves, «Jack Kerouac and the American Critics, A Select Bibliography», A:U.T. (Annales publiées trimestriellement par l'Université de Toulouse-Le Mirail, Toulouse), IX, n 1, p. 77-92.

\section{2}

Le Devoir , 28 octobre 1972, dossier consacré à l'héritage québécois et francophone de Kerouac, p. XXIX-XXXIX.

PIVANO, Fernando, «Beat, Hippie, Yippie», traduction de Jake Grassi, Paris, Christian Bourgois, 1972.

REID Malcolm, «The Shouting Signpainters: A Literary and Political Account of Québec's Revolutionary Nationalism», New York et Londres, Monthly Review Press, 1972. Reférence à Visions of Gerard.

VIDAL, Jean-Pierre et BOURQUE, Paul-André, «Le nouveau roman/Jack Kerouac», Livres et auteurs québécols, 1972, p. 202-206.

BEAULIEU, Victor-Lévy, Jack Kerouac: essai-poulet, Montréal Éditions du Jour, 1972, 235 p.

1971

BURROUGHS, William et PELIEU, Claude, Jack Kerouac: In Memoriam 1922-1969, Paris, L'Herne, coll. «Les Livres noirs», 1971.

1970

CHASSÉ, Paul-P. «Jack Kerouac: 1922-1969», Le Canado-Américain, vol. VI, $\mathrm{n}^{\circ} 1$, janvier-mars 1970 , p. 16-20.

1966

EUVRARD, Michel, «Beat, battus, et béats», Partí pris, vol. II, $\mathbf{n}^{\circ} 9$, avril 1966. Critique sur On the Road; bonne introduction à la beat generation.

\section{3}

«Interférences», in L.-F. Céline, Paris, L'Herne, 1963. Cette étude comprend un article de Kerouac sur l'écriture de Céline. Traduit par Susan Beresford. 